\title{
Ground-state degeneracy of correlated insulators with edges
}

\author{
Gil Refael ${ }^{1}$ and Hsiu-Hau Lin ${ }^{1,2,3}$ \\ ${ }^{1}$ Kavli Institute of Theoretical Physics, University of California, Santa Barbara, California 93106, USA \\ ${ }^{2}$ Department of Physics, National Tsing-Hua University, Hsinchu 300, Taiwan \\ ${ }^{3}$ Physics Division, National Center for Theoretical Sciences, Hsinchu 300, Taiwan
}

(Received 24 June 2005; published 26 August 2005)

\begin{abstract}
Using the topological flux insertion procedure, the ground-state degeneracy of an insulator on a periodic lattice with filling factor $\nu=p / q$ was found to be at least $q$-fold. Applying the same argument in a lattice with edges, we show that the degeneracy is modified by the additional edge density $\nu_{E}$ associated with the open boundaries. To carry out this generalization we demonstrate how to distinguish between bulk and edge states, and follow how an edge modifies the thermodynamic limit of Oshikawa's original argument. In particular, we also demonstrate that these edge corrections may even make an insulator with integer bulk filling degenerate.
\end{abstract}

DOI: 10.1103/PhysRevB.72.073109

PACS number(s): 71.10.Ay, 05.30.Fk, 73.43.-f, 75.30.Mb

A fascinating aspect of quantum mechanics is the interplay between global topology and some physical properties that are naively believed to depend only on local quantities. Restrictions that originate from topological arguments ${ }^{1}$ often lead to elegant and nontrivial predictions of bulk properties in a nonperturbative fashion. One example is particle statistics dictating the possible ground states of a system. A stark demonstration of that is anyons with fractional statistics. ${ }^{2}$ Another instance of this picture is the nonperturbative proof of Luttinger's theorem; ${ }^{3,4}$ it is revealed that the topology dictates the space enclosed within the Fermi surface of a Landau-Fermi liquid to be exactly the particle density in the conduction band, disregarding the details of the microscopic Hamiltonian. Topological arguments were also used to extend the Lieb-Shulz-Mattis theorem to higher dimensions. ${ }^{5}$ Beyond their elegance and appeal, topological ground-state properties have been recently made the centerpiece, primarily due to Kitaev, of a novel kind of quantum computation. ${ }^{6}$

Predictions from topological arguments are often sensitive to the geometries of the bulk. For instance, the insulating $Z_{2}$-gauge spin liquid ${ }^{7}$ is fourfold or twofold degenerate depending on whether the bulk geometry is a torus or a cylinder, respectively. Another well-known example is the $\nu=1 / q$ fractional-quantum-Hall liquid ${ }^{8}$ on the two-dimensional torus. A topological constraint due to these exotic excitations gives rise to the nontrivial $q$-fold degeneracy. Remarkably, by cutting the torus into a closed strip with two open edges, the ground-state degeneracy in the thermodynamic limit shoots up from $q$ to infinity, with gapless excitations described by the chiral Luttinger liquid on either side of the Hall bar. It is then natural to expect that the topological arguments might deliver rather different messages when edges are present. Indeed, the quantum Hall system is not the only example of juicy edge physics. Other examples are Andreev bound states appearing on edges of superconducting lattices, ${ }^{9}$ spin-1/2 excitations of the Haldane phase located at the edge of a $S=1$ Heisenberg chain, ${ }^{10}$ and the ferromagnetic moment on a zigzag tip of a carbon nanotube. ${ }^{11}$

In this Brief Report, we revisit a topological constraint in insulators - the correspondence between the filling factor and the ground-state degeneracy. In Ref. 12, Oshikawa ex- tends the Lieb-Schultz-Mattis argument ${ }^{13}$ and Laughlin's treatment of the quantized Hall conductance, ${ }^{14}$ to show that the ground-state degeneracy of an insulator with filling fraction $\nu=p / q$ (where $q$ and $p$ are coprimes) is at least $q$-fold. This is a profound statement about the degeneracy of an exact quantum ground state in the thermodynamic limit.

Using the flux insertion method on a periodic lattice, Oshikawa constructs a succession of degenerate states whose lattice momentum differs by $2 \pi[N / L]$ from one to the next, where $N$ is the particle number and $L$ the length of the system in a direction transverse to the flux. The connection to the filling fraction is inferred by taking the thermodynamic limit, where $N=\nu C L=C L p / q$ with $C=V / L$. Assuming the thermodynamic limit is taken while $C$ and $q$ are mutually prime, Oshikawa manages to construct $q$ independent states. The proof of this statement relied on the uniformity of the system in question.

Most experimental systems of relevance, however, have open boundaries. In this case, the above procedure for taking the thermodynamic limit fails, since the particle density is no longer uniform near the edges. Thus the filling factor of the bulk quantum ground state is not simply given by $N / V$. The physics of the edge is only captured through the appropriate edge filling factors that are introduced in addition to the bulk density, and the $N$ particles in the system are split between edge and bulk states. In addition, the bulk state may have a density dip near the edge. Naturally, both the bulk and edge filling factors, as well as the recession of the bulk state near the edge, determine the ground-state degeneracy in the presence of edges.

In this Brief Report we generalize the lower bound on the ground-state degeneracy to the important case of nonperiodic systems, and derive the interplay of the bulk and edge fillings in the expression for the lower bound. The distinction between bulk and edge states, as well as the definition of the edge filling fraction are not trivial. These difficulties are resolved alongside the derivation of the modified ground-state degeneracy.

Before we begin our discussion, consider a simple example. A gapped insulator on a periodic lattice made of spinless particles with bulk filling factor $\nu_{B}=1$ may have no de- 
generacy. However, if the lattice has open boundary conditions along one direction, the particle density near the edge may differ from the bulk filling factor far from the edge. This difference is captured by the edge density $\nu_{E}$, which arises from particles localized near the edge, as well as changes of the extended wave functions in the bulk. The edge state may break translational invariance and gives rise to ground-state degeneracy, even though the bulk state has perfect translational symmetry parallel to the edge. For example, for $\nu_{E}=1 / 2$ the edge may form a charge-density wave with period two. Such a state is doubly degenerate, although the bulk insulator is featureless.

Let us now review the flux insertion procedure in the context of a $d$-dimensional lattice with open boundaries in one direction, $1 \leqslant x_{1} \leqslant L_{1}$, and periodic in the remaining $d-1$ dimensions with finite length $L_{i}, i=2, \ldots, d$ (we follow Ref. 12 closely). The insulating ground state $\left|\Phi_{0}\right\rangle$ contains $N$ particles. Making use of the periodicity of the lattice, say in the $x_{d}$ direction, the ground state can be chosen to be an eigenstate of the momentum operator $\hat{P}_{d}$, carrying momentum $P_{d}^{0}$ in the $x_{d}$ direction.

We now introduce a fictitious vector potential $\vec{A}$ that couples with "unit charge" to all particles in the system. Protected by the gap, one can adiabatically insert a flux quanta $\Phi=h c$ through the ring in the $x_{d}$ direction and map $\left|\Psi_{0}\right\rangle$ to $\left|\Psi_{0}^{\prime}\right\rangle$ of the same energy. Because the flux insertion can be achieved by the constant vector potential $A_{d}=\Phi / L_{d}$ in the direction of $x_{d}$, which commutes with $\hat{P}_{d}$, the momentum remains constant in the whole adiabatic procedure, $\hat{P}_{d}\left|\Psi_{0}^{\prime}\right\rangle=P_{d}^{0}\left|\Psi_{0}^{\prime}\right\rangle$.

But the flux insertion changes the Hamiltonian from $\mathcal{H}(\Phi=0)$ to a different topological sector $\mathcal{H}(\Phi=h c)$. Before being able to compare $\left|\Psi_{0}^{\prime}\right\rangle$ to $\left|\Psi_{0}\right\rangle$, we need to restore the Hamiltonian to the same topological sector $\mathcal{H}(\Phi=0)$. This is done using the following large unitary gauge transformation:

$$
U=\exp \left(\frac{2 \pi i}{L_{d}} \sum_{\vec{x}} x_{d} \hat{n}_{\vec{x}}\right) .
$$

Now $U \mathcal{H}(h c) U^{-1}=\mathcal{H}(0)$, so $U\left|\Psi_{0}^{\prime}\right\rangle$ is an eigenket of the original Hamiltonian. Since our system is a gapped insulator, and the flux insertion was adiabatic, we expect that $U\left|\Psi_{0}^{\prime}\right\rangle$ and $\left|\Psi_{0}\right\rangle$ are degenerate [with $\mathcal{H}(0)$ in mind].

The momentum of the newfound ground state can be evaluated straightforwardly, $\hat{P}_{d} U\left|\Psi_{0}^{\prime}\right\rangle=\left(U \hat{P}_{d}+\left[\hat{P}_{d}, U\right]\right)\left|\Psi_{0}^{\prime}\right\rangle$ $=\left(P_{d}^{0}+2 \pi N / L_{d}\right) U\left|\Psi_{0}^{\prime}\right\rangle$. So the momentum of $U\left|\Psi_{0}^{\prime}\right\rangle$ is $P_{d}^{\prime}$ $=P_{d}^{0}+2 \pi N / L_{d}$. If $N$ and $L_{d}$ are mutually prime, then $U\left|\Psi_{0}^{\prime}\right\rangle$ and $\left|\Psi_{0}\right\rangle$ are two degenerate ground states. To relate the ground-state degeneracy to the filling factor, one needs to take appropriate thermodynamic limit. In a fully periodic system, Oshikawa's argument follows by saying that

$$
\Delta P=P_{d}^{\prime}-P_{d}^{0}=2 \pi \frac{N}{L_{d}}=2 \pi \nu C_{d},
$$

where $\nu=N / V=p / q$ is the filling factor of the lattice. The volume of the system is $V=\prod_{i=1}^{d} L_{i}$ and the transverse size at each particular $x_{i}$ is $C_{i}=V / L_{i}$. The thermodynamic limit is taken by fixing $\nu$ (so that $p$ and $q$ are well-defined without any correction) and choosing $C_{d}$ to be coprime with $q$. Thus, by repeating the flux insertion, we can generate $q$-fold distinct ground states. If we assume that the ground-state degeneracy $D_{g}$ is a robust quantity, disregarding the details of the thermodynamic limit, the gauge argument leads to the constraint $D_{g} \geqslant q$.

Returning to the nonperiodic system introduced above, we pick up the discussion from Eq. (2). Two different kinds of nonuniformity occur near the edges. First, the edges give rise to new states of particles localized near the edges; we define the edge-state filling factor $n_{e}=N_{E} / C_{1}$, where $N_{E}$ is the number of edge particles. Second, bulk states (forming a continuum) that propagate throughout the lattice may be repelled from the edges. This repulsion creates a charge depletion near the edge; we define $n_{\Delta}=N_{\Delta} / C_{1}$, where $N_{\Delta}$ is the total charge depleted near the edge. Both effects give rise to nontrivial corrections to the gauge argument.

Loosely speaking, the presence of edges divides the total number of particles into two groups: bulk and edge, labeled by $N_{B}$ and $N_{E}$, respectively. The precise distinction between bulk and edge states will be explained in later paragraphs. In light of this division, we need to restate Eq. (2) as

$$
\Delta P=2 \pi \frac{N_{B}+N_{E}}{L_{d}}=2 \pi \frac{N_{B}}{L_{d}}+2 \pi n_{e} C_{1 d},
$$

with $C_{1 d}=V /\left(L_{1} L_{d}\right)$. Before we can conclude anything about the ground-state degeneracy, we also need to account for the repulsion of bulk states from the edge.

As mentioned above, the edges may repel the bulk states, creating a charge depletion near the edges with density $n_{\Delta}=N_{\Delta} / C_{1}$. Now, in order to achieve the thermodynamic limit of an insulator with bulk filling $\nu_{B}$ and edge filling $n_{e}$, we need to consider a sequence of finite lattices with a total number of particles which is

$$
N_{0}=N_{B}+N_{E}=\left(\nu_{B} V-N_{\Delta}\right)+n_{e} C_{1}=\nu_{B} V+\left(n_{e}-n_{\Delta}\right) C_{1} .
$$

Note that $n_{\Delta} \equiv p_{\Delta} / q_{\Delta}$ is not necessarily an integer. To contrast, in a periodic system the thermodynamic limit would simply be taken by considering a sequence of lattices with $N_{0}=\nu_{B} V$ particles.

Including both types of edge effects, the momentum difference between $\left|\Psi_{0}\right\rangle$ and its sibling $U\left|\Psi_{0}^{\prime}\right\rangle$ is

$$
\Delta P=2 \pi \nu_{B} C_{d}+2 \pi \nu_{E} C_{1 d},
$$

with $\nu_{B}=p_{B} / q_{B}$ and $\nu_{E}=n_{e}-n_{\Delta}=p_{E} / q_{E}$. From the revised gauge argument in Eq. (5), we obtain the main result of this Brief Report: the degeneracy of an insulating state in the presence of edges is given by

$$
D_{g} \geqslant L C D\left(q_{B}, q_{E}\right) \geqslant q_{B},
$$

where $L C D$ denotes least common denominator.

Before discussing possible extensions and generalizations of the result in Eq. (6), we resolve some subtleties in the derivation above. A central issue is the difficulty of distinguishing edge and bulk particles as in Eq. (3) in a strongly correlated system, where the concept of single particle states is rather vague. At first sight, this distinction may seem impossible to carry out in practice, and might be mistaken to be 
a theorist's whim; there is, however, a precise way of counting the number of edge and bulk states. This method utilizes a spectral function decomposition of the system's correlation function, which in principle we know with utmost precision. Following Lehman decomposition of the single-particle spectral function:

$$
\begin{aligned}
A(\vec{x}, \omega)= & \sum_{m} \delta\left(\omega-E_{m}\right)\left|\left\langle m\left|\psi^{\dagger}(\vec{x})\right| \Psi_{0}\right\rangle\right|^{2} \\
& \pm \sum_{m} \delta\left(\omega+E_{m}\right)\left|\left\langle m|\psi(\vec{x})| \Psi_{0}\right\rangle\right|^{2} .
\end{aligned}
$$

Here $\psi(\vec{x})$ is the annihilation operator in the Schrödinger picture at lattice site $\vec{x}$. The \pm distinguishes between fermions and bosons. Without loss of generality, let us concentrate on fermions. Note that $|m\rangle$ are many-body excited states that show one particle excitations. The excitations correspond to one-particle states of free particles. In the following we simply refer to $|m\rangle$ as states.

Next, consider the $\omega<0$ (hole) part of the spectral function $A(\vec{x}, \omega)$. In a finite system, it is given as a discrete sum of $\delta$-functions multiplied by matrix elements, and it obeys the sum rule

$$
\sum_{\vec{x}} \int_{-\infty}^{0} A(\vec{x}, \omega) d \omega=N,
$$

where $N$ is the total number of particles in the lattice. If we take the limit $L_{1} \rightarrow \infty$ while keeping the bulk density fixed, we expect that some of the $\delta$-functions will merge into continuum, whereas the rest will remain sharp and separated. The first group is the excited bulk states, and the second group is the excited edge states. We stress that $|m\rangle$ are strongly correlated many-body states that show one particle excitations $\Delta Q= \pm 1$ relative to the ground state. More precisely, bulk states $\left|m_{B}\right\rangle$ scale as

$$
\left|\left\langle m_{B}\left|\psi^{\dagger}(\vec{x})\right| \Psi_{0}\right\rangle\right|^{2} \sim \mathcal{O}\left(\frac{1}{L_{1}}\right)
$$

for any $\vec{x}$, as $L_{1}$ is scaled to infinity. Unlike bulk states, the edge states, $\left|m_{E}\right\rangle$, tend to an $\vec{x}$-dependent constant as $L_{1}$ $\rightarrow \infty$ :

$$
\left|\left\langle m_{E}\left|\psi^{\dagger}(\vec{x})\right| \Psi_{0}\right\rangle\right|^{2} \sim \mathcal{O}(1) .
$$

Thus we can break the spectral function into bulk $\left(A_{B}\right)$ and edge $\left(A_{E}\right)$ parts:

$$
\begin{aligned}
A_{B / E}(\vec{x}, \omega)= & \sum_{m_{B / E}} \delta\left(\omega-E_{m_{B / E}}\right)\left|\left\langle m_{B / E}\left|\psi^{\dagger}(\vec{x})\right| \Psi_{0}\right\rangle\right|^{2} \\
& \pm \sum_{m_{B / E}} \delta\left(\omega+E_{m_{B / E}}\right)\left|\left\langle m_{B / E}|\psi(\vec{x})| \Psi_{0}\right\rangle\right|^{2} .
\end{aligned}
$$

The definition of $N_{B}$ and $N_{E}$ is hence

$$
N_{B(E)}=\sum_{\vec{x}} \int_{-\infty}^{0} d \omega A_{B(E)}(\vec{x}, \omega) .
$$

There are two subtleties in the above procedure. First, it is possible that an edge state hybridizes with a bulk state. Definition along the lines of Eqs. (11) and (12) specifies that such a state is an edge state; even though such a state is partially delocalized, it is associated with the edge due to the localized weight. The number of such states will scale with $L_{i}$ for $i>2$, but not with $L_{1}$. Another subtlety occurs if there is an accidental degeneracy between a bulk and edge states. In this case, in a finite system, it may be impossible to distinguish between the edge and bulk states and two hybridized states. This accidental degeneracy will surely be lifted by a different choice of system dimensions, and therefore needs not bother us.

Now that the derivation is complete, we would like to elaborate on some extensions of the edge argument. The first question we address is the effect of bulk-state depletion near the edge. Note that if $n_{\Delta}$ is an integer, the bound on the ground-state degeneracy, $D_{g} \geqslant L C D\left(q_{B}, q_{e}\right)$, only depends on $\nu_{B}$ and $n_{e}=p_{e} / q_{e}$. In this case, the depletion of bulk states does not play any role in determining ground-state degeneracy. In fact, for featureless insulators with low-energy excitations described by Fermi liquid theory, we can show that $n_{\Delta}$ is an integer.

Start with the ground state $|0\rangle$ of the featureless insulator on a periodic lattice (without edges). The insulator with an open edge can be viewed as the original ground state $|0\rangle$, but with hole excitations near the boundary. Translational invariance ensures that we can construct local quasihole states $|r\rangle=\psi(r)|0\rangle$, with the same spatial profile but located at different $C_{1}$ lattice sites on the edge. In general, these states, located at different lattice sites, would have nonvanishing overlaps. If one assumes the Fermi-liquid picture is at work here, these local (Wannier) orbitals would form a continuous band. To ensure the new ground state (in the presence of open boundaries) is also insulating, these quasiparticles must form a band insulator at the edge, i.e., the number of holes in one unit cell, $n_{\Delta}=N_{\Delta} / C_{1}$, is an integer.

The argument also applies to the simple but exotic situation where the low-energy excitations carry fractional "charges" (not necessarily the ordinary electric charge), but are well-described by a Fermi-liquid-like theory. By a similar argument to the one above, $n_{\Delta}$ is fractional, reflecting the breaking up of ordinary particles. Although the argument does not hold when low-energy excitations are not described by Fermi liquid theory, $n_{\Delta}$ may still serve as a useful precursor for identifying low-energy excitations in featureless insulators.

Another instance of the theorem is in the case where the edges are far apart. It is then natural to then associate the edge corrections with the different edges enumerated by $i$ : $n_{e}=\sum_{i} n_{e i}$ and $n_{\Delta}=\sum_{i} n_{\Delta i}$. A simple example helps to demonstrate this situation and the statements above. Consider a segment of carbon nanotube with circumference $L_{y}$ (in the $y$ direction) and zigzag edge at $x=0$ and $x=L_{x}$. Choose the tight-binding hopping to be in the strong anisotropic regime, $\left|t_{h}\right|>2|t|$, where $t_{h}$ and $t$ denote the hopping amplitudes along horizontal and (vertical) zigzag bonds. The interactions between particles are assumed to be weak, with a typical energy scale $V$.

Ignoring the weak interaction momentarily, the open boundaries at $x=0$ and $x=L_{x}$, give rise to single-particle edge states at $E=0$ with momentum-dependent localization length $\xi\left(k_{y}\right)=\ln \left|\left(2 t \cos k_{y}\right) / t_{h}\right|$. Each edge has $L_{y}$ distinct states, cor- 
responding to each quantized $k_{y}$. Since the bare hopping Hamiltonian is quadratic, the total number of states has to equal the number of particles in the lattice, or twice the number of unit cells (each carbon-nanotube unit cell contains two sites). Hence the $L_{y}$ edge states must come at the expense of $L_{y}$ bulk states. This leads to $n_{\Delta}=1$ at natural filling $\nu_{B}=1$, and thus there is no effect on the degeneracy due to the bulk states repulsion at the edge.

Nevertheless, the edge-state filling factor, $n_{e}=n_{e 1}+n_{e 2}$, may be tuned to arbitrary fractions by removing or adding a small amount of electrons (proportional to the length $L_{y}$ ) which do not affect $\nu_{B}$. Because the interaction $V$ is weak (compared with the bulk gap $\Delta_{B}$ ), the nontrivial mixing of single-particle states occurs within edge states and lifts the exact degeneracy at $E=0$. The ground state is nontrivial and certainly depends on the specific form of interactions. But the revised gauge argument with the momentum shift in Eq. (5) leads to at least $q_{e}$-fold degeneracy of the ground state even though the bulk filling is an integer.

When considering particular physical systems, as in the above example, it is possible that the ground-state degeneracy will be larger than our rigorous result in Eq. (6). If the two edges are known by other means to be independent (as is probably the case in many large systems) and are also independent of the bulk state, then the degeneracy should be at least the product $q_{e 1} q_{e 2} L C D\left(q_{\Delta}, q_{B}\right)$. This degeneracy would become the number of low-lying states if there is a weak interaction between the two edges. Other cases are also possible, most notably, independence of the edge states from the bulk, but not from each other. In this case the degeneracy is $\operatorname{LCD}\left(q_{e 1}, q_{e 2}\right) \cdot \operatorname{LCD}\left(q_{\Delta}, q_{B}\right)$. We add that for incommensurate edge filling in the thermodynamic limit $L \rightarrow \infty$, it is very likely that the low-energy physics of the system is described by a gapless liquid on the edge, living inside the gapped bulk spectrum (similar to the chiral edge states in quantum Hall liquid). Since these edge excitations sometimes are the only low-energy excitations, appropriate treatment of them is crucially important.

An interesting and perhaps common situation is when the bulk and edges conspire to produce a uniform particle density near the edge. This happens if the bulk states repelled from the edge are exactly compensated by particles trapped at the edge: $n_{e}=n_{\Delta}$. Our revised gauge argument is then reduced to Oshikawa's original argument with only the bulk filling determining the degeneracy.

The revised gauge argument can be generalized to the case of more than one nonperiodic direction. We require that at least one of the directions of the lattice is periodic to derive a generalized gauge argument, although this restriction may not be necessary in a physical system. We consider $x_{d}$ as periodic. If all other sides of the lattice terminate at $x_{i}=0$ and $x_{i}=L$, there may be edge (surface) and wedge states, with filling numbers that scale as $N_{\alpha} \sim L^{\alpha}$, where $\alpha=1,2, \ldots, d-1$. One then needs more filling factors $n_{\alpha}$ to specify the filling of the system. Also, one needs the depletion parameters $n_{\Delta a}^{b}$ where $a=1, \ldots, d$ specifies the repelled "bulk" state, and $b=1, \ldots, a-1$ is the number of restricted dimensions for the bulk states. To clarify this statement consider the example of a cube that is made periodic in the $z$ direction. The bulk is three-dimensional, and the depletion away from the surfaces $(x=0, x=L, y=0$, and $y=L)$ amounts to $n_{\Delta 3}^{1} L^{2}$ particles. Also, the wedges at $x=0, y=0, x=0, y$ $=L$, etc. may repel the bulk states with a volume of $n_{\Delta 3}^{2} L$. Similarly, edge (surface) states on the faces of the cube may be repelled from the wedges with corrections $n_{\Delta 2}^{1} L$.

In summary, using the topological flux insertion procedure, we rederive the connection between the ground-state degeneracy in an insulator with its filling factors $\nu_{B}$ in the bulk and $\nu_{E}$ at the edge. As expected, the presence of open edges produces rich physical phenomena at the boundaries. In addition, the response of the bulk states to the edge may lend a different perspective into the correlated bulk physics.

We would like to thank Leon Balents, Matthew Fisher, Arun Paramekanti, Tami Pereg-Barnea, and particularly Masaki Oshikawa for insightful discussions. H.H.L. is grateful for support from NSC-91-2120-M-007-001 and NSC-922112-M-007-039. G.R. thankfully acknowledges support from NSF fund PHY99-07949.
${ }^{1}$ X. G. Wen, Adv. Phys. 44, 405 (1995).

${ }^{2}$ Y.-H. Chen, F. Wilczek, E. Witten, and B. I. Halperin, Int. J. Mod. Phys. B 3, 1001 (1989).

${ }^{3}$ M. Oshikawa, Phys. Rev. Lett. 84, 3370 (2000).

${ }^{4}$ A. Paramekanti and A. Vishwanath, Phys. Rev. B 70, 245118 (2004).

${ }^{5}$ M. B. Hastings, Phys. Rev. B 69, 104431 (2004).

${ }^{6}$ A. Yu. Kitaev, Ann. Phys. (San Diego) 303, 2 (2003).

${ }^{7}$ T. Senthil and M. P. A. Fisher, Phys. Rev. B 62, 7850 (2000).
${ }^{8}$ W.-G. Wen, Int. J. Mod. Phys. B 6, 1711 (1992).

${ }^{9}$ C.-R. Hu, Phys. Rev. Lett. 72, 1526 (1994).

${ }^{10}$ P. P. Mitra, B. I. Halperin, and I. Affleck, Phys. Rev. B 45, 5299 (1992).

${ }^{11}$ G. Refael, H.-H. Lin, and M. Oshikawa (unpublished).

${ }^{12}$ M. Oshikawa, Phys. Rev. Lett. 84, 1535 (2000).

${ }^{13}$ E. H. Lieb, T. Schultz, and D. J. Mattis, Ann. Phys. (N.Y.) 16, 407 (1961).

${ }^{14}$ R. B. Laughlin, Phys. Rev. B 23, 5632 (1981). 